\title{
Diaphragm Dysfunction (Paralysis) in a Cystic Fibrosis Patient
}

\author{
Zubair Khan $\mathbf{M}^{1 *}$, Chaudary $\mathbf{N}^{2}$, Abraham $\mathbf{A}^{1}$ and \\ Patel K ${ }^{1}$ \\ ${ }^{1}$ Department of Internal Medicine, St Mary Medical \\ Center, USA \\ ${ }^{2}$ Virginia Commonwealth University Hospital, USA \\ *Corresponding author: Muhamamd Zubair Khan, \\ Department of Internal Medicine, St Mary Medical \\ Center, 1201 Langhorne-Newtown Rd, Langhorne, PA, \\ 19047, USA
}

Received: December 24, 2020; Accepted: J anuary 04, 2021; Published: January 11, 2021

\begin{abstract}
Diaphragmatic Paralysis (DP) is a rare disease and usually occurs secondary to systemic processes, although idiopathic forms have been described. Management of diaphragmatic paralysis depends on multiple factors, including the etiology, presence, or absence of symptoms, and more importantly, the presence of nocturnal hypoventilation. DP has been described in both unilateral and bilateral forms. In unilateral DP, most patients are asymptomatic and often do not require intervention. Symptomatic patients with unilateral DP, however, will require plication. In bilateral DP, phrenic nerve pacing or nasal Continuous Positive Airway Pressure, Bilateral Positive Airway Pressure (CPAP BiPAP), or intermittent positive pressure ventilation by the nasal or oral mask is recommended. We describe a case of unilateral DP in a cystic fibrosis patient treated by surgical plication.
\end{abstract}

Keywords: Diaphragmatic paralysis, Cystic fibrosis

\section{Introduction}

Cystic Fibrosis (CF) is the most common lethal inherited disease in Caucasian descent [1]. CF is an autosomal recessive disorder, and most CF carriers are asymptomatic. In CF patients, gastrointestinal, pulmonary, and genitourinary systems are most commonly affected, and patients frequently have symptoms related to any of these affected systems. To the best of our knowledge, the involvement of skeletal muscles such as the diaphragm has rarely been described in CF cases. Our case describes the etiology and management of diaphragmatic dysfunction in a CF patient.

\section{Case Report}

A twenty-three-year-old male with a past medical history of CF, Gastroesophageal Reflux (GERD), inguinal hernia, hiatal hernia, ventral hernia, history of chronic Pseudomonas aeruginosa, and Mycobacterium abscesses presented to the hospital with $\mathrm{CF}$ exacerbation. He was admitted for his pulmonary symptoms, and IV antibiotics were started. PFTs (FEV1\%) were decreased from his baseline. Chest X-ray and non-contrast CT revealed progressive bronchiectasis and volume loss of both lungs, with right-sided predominance (Figure 1). During hospitalization, performance on PFTs declined despite the radiographic suggestion of improvement. He was discharged after a 6-week hospitalization and at the 4-week clinic follow up he reported worsening shortness of breath. As an outpatient, his PFTs did not show improvement despite IV antibiotic therapy with amikacin. He was re-admitted and placed on a new regimen of IV antibiotics, including imipenem-cilastatin, amikacin, cefoxitin, tigecycline, and oral antibiotics doxycycline and azithromycin. CBC revealed new leukocytosis, and a new chest X-ray showed elevation of the right hemidiaphragm. A repeat chest CT confirmed the right hemidiaphragm's significant elevation with progressive fibrosis and volume loss of right and left lungs without bronchial obstruction. He underwent bronchoscopy and Broncho-Alveolar Lavage (BAL), which confirmed patent airways. BAL cultures subsequently grew

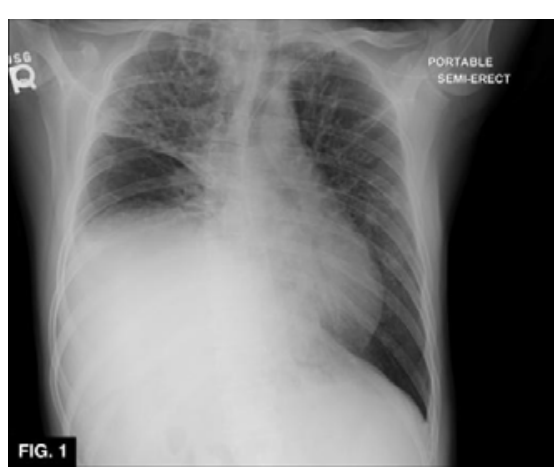

Figure 1: Chest X-ray showed progressive bronchiectasis and volume loss of both lungs, with right-sided predominance as compare to left sided.

Mycobacterium. Sniff testing using fluoroscopic guidance confirmed the paralyzed right hemidiaphragm. The results of the studies were felt to be consistent with idiopathic unilateral Diaphragmatic Paralysis (DP). Multi-disciplinary discussions and consideration led to the decision to pursue surgical plication. The patient underwent right thoracotomy and plication of the right hemidiaphragm by cardiothoracic surgery and was continued on the aforementioned intravenous antibiotic regimen. He tolerated the surgery well, and his PFTs began showing trends toward improvement. At follow-up, six weeks later, he had significant improvement in his shortness of breath, subjective quality of life, and exercise capacity. His follow up chest X-ray showed significant improvement (Figure 2).

\section{Discussion}

Several studies have reported many causes and risk factors that compromise the diaphragm function, but there has been no report of CF's association with diaphragmatic paralysis [2].

Despite significant advancement in CF therapy, recurrent infections, and inflammation in the lungs cause progressive lung
Austin J Womens Health - Volume 8 Issue 1 - 2021

Submit your Manuscript | www.austinpublishinggroup.com

Zubair Khan et al. (C) All rights are reserved
Citation: Zubair Khan M, Chaudary N, Abraham A and Patel K. Diaphragm Dysfunction (Paralysis) in a Cystic Fibrosis Patient. Austin J Womens Health. 2021; 8(1): 1044. 


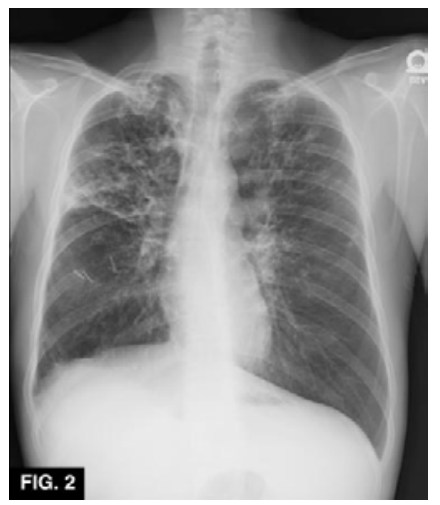

Figure 2: CXR after right thoracotomy and plication of right hemi-diaphragm showed significant improvement in volume of lung.

function loss. Over time, the respiratory muscles cannot sustain the increased breathing requirements, which often leads to respiratory failure. Logically, generalized skeletal (diaphragm) muscle atrophy and weakness have been described in CF patients. This can be due to many factors, most notably the mechanical limitations of respiratory muscles and malnutrition.

Divangahi et al. studied mice with a lack of the CF Transmembrane Conductance Regulator (CFTR) and determined that CFTR plays an intrinsic role in skeletal muscle atrophy and dysfunction. The CFTR protein is located at the sarcoplasmic reticulum in the diaphragm's skeletal muscle fibers, regulating intracellular calcium fluxes [3]. The defective CFTR in the CF mice is associated with increased Ca mobilization from the sarcoplasmic reticulum, increasing the intracellular calcium. As a result, both contractility and relaxation of the diaphragm are impaired. Dysfunction of the CFTR protein also upregulates pro-inflammatory gene expression. These proinflammatory genes are activated during lung infections, increasing the inflammatory mediators, which activate cytokines and ubiquitin ligase pathways, causing degradation of protein in the diaphragm and ultimately diaphragmatic dysfunction. These two mechanisms are the main reason for diaphragmatic dysfunction in the defective CFTR cell. In this case, the diaphragm dysfunction is most likely secondary to CFTR protein dysfunction [2]. Our patient was also taking aminoglycosides, which can predispose to diaphragmatic dysfunction [3].

Management of diaphragmatic paralysis depends upon multiple factors: etiology, unilateral vs bilateral involvement, and the presence or absence of symptoms. Patients with unilateral diaphragmatic paralysis are usually asymptomatic and frequently do not require treatment. However, some patients with unilateral diaphragmatic paralysis have dyspnea symptoms due to the presence of superimposed pulmonary disease or to the increased ventilatory demands of intense physical activity. Surgical plication is generally the best treatment in such cases. Plication may be performed using thoracoscopic, laparoscopic, or open approaches.
In some cases, patients with unilateral diaphragmatic paralysis will develop acute respiratory insufficiency. In such cases, either invasive mechanical ventilation or Noninvasive Positive Pressure Ventilation (NPPV) is the best option. Patients can usually be weaned from ventilatory support once the acute illness is resolved [4].

Bilateral diaphragmatic paralysis usually has symptoms of dyspnea and orthopnea. In such cases, Noninvasive Positive Pressure Ventilation (NPPV) is initiated in these patients using a Bi-level Positive Airway Pressure device (BiPAP). Diaphragmatic pacing can also be used to wean patients from or delay the need for ventilatory support. Phrenic nerve pacing can provide full ventilatory support for ventilator-dependent patients who have bilateral diaphragmatic paralysis and intact phrenic nerves [5]. In our case, the patient had unilateral diaphragmatic paralysis in the setting of both worsening pulmonary function testing as well as significant dyspnea. Surgical plication was performed with subsequent improvement in both pulmonary function testing and symptoms.

\section{Conclusion}

To our knowledge, this is a rare case in which diaphragmatic dysfunction has been reported in a CF patient. The management of unilateral diaphragmatic dysfunction in CF patients is different from other patients because of compromised lung function intrinsic to CF. Such patients are more likely to be symptomatic, and surgical plication using thoracoscopic and laparoscopic approaches is the best option. Also, aminoglycosides have been reported as a known predisposing factor for diaphragmatic paralysis, and most CF patients, incidentally, receive aminoglycoside therapy. In the setting of diaphragmatic dysfunction, aminoglycosides should be used with extreme caution.

\section{References}

1. Davis PB, Drumm M, Konstan MW. Cystic fibrosis. Am J RespirCrit Care Med. 1996; 154: 1229-1256.

2. Wilcox PG, Pardy RL. Diaphragmatic weakness and paralysis. Lung. 1989; 167: 323-341.

3. Divangahi M, Balghi H, Danialou G, Comtois AS, Demoule A, Ernest S, et al. Lack of CFTR in skeletal muscle predisposes to muscle wasting and diaphragm muscle pump failure in cystic fibrosis mice. PLoS Genet. 2009; 5: 1000586.

4. Dubé BP, Dres M. Diaphragm Dysfunction: Diagnostic Approaches and Management Strategies. J Clin Med. 2016; 5: 113.

5. Mills GH, Kyroussis D, Hamnegard CH, Polkey MI, Green M, Moxham J. Bilateral magnetic stimulation of the phrenic nerves from an anterolateral approach. Am J RespirCrit Care Med. 1996; 154: 1099-1105. 